\title{
Clinical and Radiographic Outcomes of Vital Inflamed Pulp Therapy in Immature Permanent Teeth with Irreversible Pulpitis: A Retrospective Case Series
}

\section{Wen Xiao}

Shanghai Jiao Tong University School of Medicine

\section{Wentao Shi}

Shanghai Jiao Tong University School of Medicine

Jun Wang ( $\nabla$ wangjun202@126.com )

Shanghai Ninth People's Hospital, College of Stomatology, Shanghai Jiao Tong University School of Medicine

\section{Research article}

Keywords: Retrospective study, immature teeth, vital pulp therapy, apical periodontitis, radiographic outcomes

Posted Date: September 10th, 2020

DOI: https://doi.org/10.21203/rs.3.rs-52921/v1

License: (c) (1) This work is licensed under a Creative Commons Attribution 4.0 International License. Read Full License 


\section{Abstract}

\section{Background}

To assess the resolution of clinical symptoms and radiographic changes in root length and apical diameter in immature permanent teeth with irreversible pulpitis or apical periodontitis with vital inflamed pulp therapy (VIPT).

\section{Methods}

The faculty members at the Ninth People's Hospital pediatric dentistry department were invited to submit consecutive VIPT cases treated by them, irrespective of outcome, between 2014 and 2016. Clinical success rate, radiographic changes in periapical radiolucency, and apical closure were analyzed, and radiographic changes in the apical diameter and root length were quantified.

\section{Results}

Thirteen of 14 submitted cases of irreversible pulpitis in immature teeth met the inclusion criteria. The follow-up period ranged from 17 to 37 (average, $26.5 \pm 7$ ) months. All 13 treated teeth (100\%) survived and $12(92.3 \%)$ met the clinical criteria for success throughout the follow-up period, with $92.3 \%$ of cases (12 of 13) showing a significant periapical radiolucency decrease and 84.6\% (11 of 13) showing complete apical closure at the last visit. The change in apical diameter and root length were obvious.

\section{Conclusions}

Vital inflamed pulp therapy approaches might be of particular value in restoring root development and apical closure and can be an option in treating immature teeth with irreversible pulpitis, even apical periodontitis.

\section{Background}

A vital pulp is a prerequisite for immature permanent tooth development. However, trauma, caries, developmental malformations, and other factors often lead to pulpal lesions of immature permanent teeth.

It used to be generally accepted that a history of spontaneous or lingering provoked pain, swelling, or periapical radiolucency was indicative of extensive, irreversible inflammatory changes of the pulp tissue. These changes meant that the pulpal inflammation had reached a level at which its elimination was not possible without removal of the entire pulp. Thus, radical treatment such as apexification, apical barrier surgery, or regenerative endodontic treatment (RET) should be performed for immature teeth [1]. Although an apical barrier can be established by apexification and apical barrier surgery, they do not help restore a functional pulp-dentin complex and do not allow further root development, leaving the tooth susceptible to fracture [2-5]. In recent studies, RET provided a new treatment modality for the previously described 
cases. There have been many successful case reports and clinical trials published [6-9]. However, the treatment is complicated [10], and its outcome unpredictable [11].

It has been found that clinical diagnosis based on pain quality, history, and swelling or periapical radiolucency has a low correlation with pulp status in situ [12]. Therefore, it is doubtful that radical treatment should be performed for teeth only by clinical diagnosis [13]. Moreover, it is reported that before the infection involved the entire dental pulp, there were inflammatory changes around the apical area [14]. But there were still residual nerve fibers in the pulp even if the pulp infection was serious or partially necrotic [14], and there were still residual vital pulp tissues in the pulp cavity even in immature permanent teeth with periapical periodontitis and bone destruction [15]. The surviving cells had the potential to resume proliferation and differentiation after eliminating the inflammation and restoring pulp function [15]. Additionally, compared with the mature teeth, the pulp tissue of immature permanent teeth has a rich blood supply and high cell composition [12]. All these events make it seem reasonable to conserve the residual vital pulp tissue and eliminate the infection to induce root development, indeed, this has been verified by many case reports.

Ronald et al.[16, 17] reported that during root canal exploration, the following conditions appear, indicating that there is residual vital pulp tissue in the root canals of immature permanent teeth with irreversible pulpitis, even with apical periodontitis: (1) in cases when inserting a file or gutta-percha cone into the canal, a little resistance, caused by the presence of tissue, is felt; (2) the patient reports a sensation of pain; (3) the patient still feels uncomfortable even under local anesthesia. Treatment to conserve residual vital pulp tissues was consistent with the hypothesis of a functional restoration of biologic root development, so it was defined as Vital inflamed pulp therapy (VIPT) [17]. In previous case reports, the researchers [17-21] all achieved good prognosis of clinical symptom regression, apical closure, and even continued root development.

Case series suggest that Vital inflamed pulp therapy approaches might be of particular value in restoring root development and apical closure in some difficult cases but lack quantifying analysis. The purpose of this retrospective study is to evaluate the clinical and radiographic changes and quantify those radiographic changes in patients receiving VIPT for immature permanent teeth with irreversible pulpitis, even with apical periodontitis.

\section{Materials And Methods}

\section{Sample Selection}

This study received approval from the Office of Human Research Ethics at the Ninth People's Hospital, Shanghai Jiao Tong University, School of Medicine (Ref: SH9H-2019-T350-1). Our research has been conducted in full accordance with the World Medical Association Declaration of Helsinki.

The Ninth People's Hospital Department of Paediatric Dentistry has a prescribed methodology for VIPT, and every faculty member has been trained in standard operating procedure. The faculty were invited to 
submit consecutive VIPT cases treated by them, irrespective of outcome, during the period June 2014 to June 2016. The faculty members were asked to fill out the clinical records data with radiographic and other examination notes for submission; patient's personal information was masked.

Each case that fulfilled the following criteria was included in the outcome analysis: (1) children in good general health with no systemic diseases and aged 7-12 at the time of treatment; (2) immature permanent teeth (stage 7-9 according to Nolla's criteria[22]) diagnosed as irreversible pulpitis with or without periapical pathology at the initial appointment; (3) the immature teeth were treated with VIPT and the final restoration placed; (4) patients were followed up for at least 12 months and their records were complete.

\section{Data Extraction}

Cases identified that met the inclusion criteria were assigned a research subject number, and data were extracted from their clinical record, and calibration exercises were blinded to the research purpose. Data collection was accomplished using a standardized electronic form, including history (patient's sex, age, tooth number, Nolla's stage), etiology, preoperative signs and symptoms, presence or absence of periapical radiolucency (PARL), treatment, and follow-up periods. Details of the clinical procedure and any variation were also recorded, and the preoperative radiographs were checked for immature apices.

The final data set was exported to Excel and saved on a secure server for analysis. All radiographs related to study cases were collected and stored in a digital format on a secure server.

\section{Clinical Protocol}

The operators completed all the study procedures at the Department of Paediatric Dentistry, Ninth People's Hospital, Shanghai Jiao Tong University, School of Medicine, China. The treatment was completed in two or three visits. At the first appointment, parallel periapical x-ray films were taken before the operation to examine the periapical tissue and root development. The protocol consisted of local anesthesia (lidocaine $2 \%$ without adrenaline), rubber dam isolation, gaining access to the pulp chamber, exploring the vital pulp tissue in the root canal with $25 \# \mathrm{~K}$-file and recording the tissue's depth, and irrigating with copious amounts of $2.5 \% \mathrm{NaOCl}, 3 \%$ hydrogen peroxide solution, and $0.9 \%$ sterile saline solution (NS) without instrumentation (NS as last irrigation). After irrigating, the root canal was dried with sterile paper points, calcium hydroxide paste (Ninth People's Hospital, Shanghai, China) was placed in the root canal, and the cavity was sealed with glass ionomer cement (Glaslonomer FX-II; Shofu Inc, Kyoto, Japan). Two weeks later, if clinical symptoms persisted, the initial treatment procedures were repeated. Otherwise, the tooth was isolated with a rubber dam for the next treatment. After local anesthesia and removal of the temporary restoration, the canal was irrigated with copious amounts of $2.5 \% \mathrm{NaOCl}$ and $3 \%$ hydrogen peroxide solution and $0.9 \%$ NS (NS as last irrigation) and dried with sterile points. Calcium hydroxide paste or MTA (Pro-Root MTA White; Densply International, Inc, Konstanz, Germany) or i-Root BP 
Plus (Innovative BioCeramix Inc, Burnaby, Canada) was placed over the residual vital pulp tissue and covered by conventional glass ionomer cement and resin composite (Z350; 3M ESPE, St Paul, Minn., USA) were used to fill the access cavity. The patient was followed up at 3,6 , and 12 months, then at regular 6-month intervals after completion of VIPT. Follow-up included clinical assessment of pain or discomfort, swelling, sinus tract, mobility, and acquisition of a periapical radiograph.

\section{Clinical Outcomes}

Tooth survival and clinical success were evaluated as clinical outcomes [23]. Tooth survival was defined as the tooth's remaining in the arch throughout the study period. While clinical success was defined as a tooth that survived and without any clinical assessment during the recall period [24].

\section{Radiographic Outcomes and Analysis}

Study investigators who were blinded to the research purpose evaluated the preoperative and postoperative radiographs. The records included the presence or absence of PARL and root development changes (the changes in apical foramen width and root length). The changes in apical foramen width and root length were measured from the preoperative and postoperative images. The periapical X-ray films should be taken by the paralleling technique and standardization performed by using TurboReg (Biomedical Imaging Group, Swiss Federal Institute of Technology, Switzerland) [24], better than CBCT (cone-beam computed tomography) for the consideration of human ethics. For root length, the measurements were done on a straight line from the CEJ to the midpoint of the radiographic apex from both mesial and distal, while the apical diameter was measured as a straight line across the radiographic apical foramen. Then the results were measured by using NIH Image $\mathrm{J}$ (version 1.41; National Institutes of Health, USA). We present the data as percentage change from preoperative values rather than the actual millimetric data to eliminate one potential source of systematic error in the overall analysis of treatment outcome [25]. The same investigator collected all the measurements and repeated after 2 weeks, and the mean of the two replicates was reported as the final value.

\section{Statistical Analysis}

The Fisher test was used to compare the proportion of cases with or without PARL present and closure of the apical foramen at the 3-, 6-, and 12-month follow-ups and the last recall time versus the baseline proportion of cases. While the changes in root length and apical diameter were expressed as mean \pm standard error of mean. The data were summarized and analyzed by professional statistician using SPSS 13.0 statistical software (SPSS Inc, Chicago, USA) and $P<0.05$ was regarded as statistically significant. Graphs were generated by using GraphPad Prism 8 (GraphPad Software Inc, Calif, USA).

\section{Results}




\section{Baseline Characteristics of the Study Population}

Fourteen cases were treated and submitted by the faculty during the previously stated treatment duration. A total of 13 immature teeth out of 14 (in 13 patients) between the ages of 7 and 12 years met the inclusion criteria. One case was excluded because of lack of a detailed description of the treatment protocol.

Of the 13 cases (including three incisors, five premolars, and five molars), etiology of the teeth included traumatic (one tooth), anatomic (seven teeth), and carious (five teeth). All treated teeth had preoperative clinical symptoms with necrotic dental pulps, $84.6 \%$ (11 of 13) of which had periapical symptoms (11/13 subjects reported sensitivity to percussion, while $9 / 13$ had a sinus tract) and radiographic periapical pathology. The demographics and baseline clinical data of the patients are collected in Table 1. 
Table 1

Patient Demographics and Baseline Characteristics

\begin{tabular}{|c|c|c|}
\hline \multirow[b]{2}{*}{ Variable } & \multicolumn{2}{|c|}{ Vital inflamed pulp therapy $(\mathrm{N}=13)$} \\
\hline & Teeth(N) & $\%$ \\
\hline \multicolumn{3}{|l|}{ Sex } \\
\hline Female & 7 & 53.85 \\
\hline Male & 6 & 46.15 \\
\hline Age-years & $9.9 \pm 1.5$ & \\
\hline Follow up Period-Months & $26.5 \pm 7$ & \\
\hline \multicolumn{3}{|l|}{ Tooth type } \\
\hline Incisor & 3 & 23.08 \\
\hline Premolar & 5 & 38.46 \\
\hline Molar & 5 & 38.46 \\
\hline \multicolumn{3}{|l|}{ Etiology } \\
\hline Trauma & 1 & 7.69 \\
\hline Anatomical & 7 & 53.85 \\
\hline Caries & 5 & 38.46 \\
\hline \multicolumn{3}{|c|}{ Stage of Root Development (Nolla) } \\
\hline 7 & 4 & 30.77 \\
\hline 8 & 3 & 23.08 \\
\hline 9 & 6 & 46.15 \\
\hline \multicolumn{3}{|l|}{ Signs and symptoms } \\
\hline Absent & 0 & 0.00 \\
\hline Present & 13 & 100.00 \\
\hline \multicolumn{3}{|l|}{ Apical periodontitis } \\
\hline Absent & 2 & 15.38 \\
\hline Present & 11 & 84.62 \\
\hline
\end{tabular}




\section{Clinical Outcomes}

The cases were followed from 17 to 37 (average, $26.5 \pm 7$ ) months, with a review at 3,6 , and 12 months, then once every 6 months. All 13 teeth survived, and 92.3\% (12 of 13) met the study criteria for clinical success at 12 months. Representative cases are presented in Figures $\mathbf{1} \underline{\mathbf{3}}$.

\section{Radiographic Outcomes}

92.3\% of cases (12 of 13) showed no periapical radiolucency (PARL) at the 12-month follow-up and $84.6 \%$ of cases (11 of 13) exhibited complete radiographic apical closure at the last post-treatment visit (Figure 4). The changes in apical diameter and root length were obvious. In apical diameter averaged decreasing $7.98 \%$ at 3 months, $70.3 \%$ at 12 months, and $91.0 \%$ at the last review, while in root length averaged increasing $11.4 \%$ at 3 months, $27.4 \%$ at 12 months and $33.36 \%$ at the last review (Figure $\underline{\mathbf{5}}$ ). At the last review, 12 patients had achieved clinically meaningful change for apical diameter measurements, and five had met this criterion for root length measurements [23].

\section{Discussion}

Although several case presentations have reported the clinical and/or radiographic outcomes of VIPT [1721], to our knowledge, this is the first retrospective study to observe the quantification of clinical and radiographic changes. This study included all the tooth types and Nolla's 7-9 stage of root development. The etiology of the teeth in this study included traumatic, anatomic, and carious (including all the etiologies for immature permanent teeth with irreversible pulp infection or periapical infection), so the result of clinical and radiographic changes of VIPT is persuasive. The results will be compared with the contemporary treatment in immature permanent teeth with irreversible pulp infection or periapical infection, RET and apexification. In addition, in this study, all dentists were trained in standard operating procedure to control the case-to-case variability.

In this study, we analyzed the outcome of clinical symptoms and radiographic healing of irreversible pulpitis and apical periodontitis. Then the radiographic signs of further root development were quantitated, including apical diameter and root length. All the teeth survived; $92.3 \%$ of our cases $(12$ of 13) showed resolution of periapical lesions and $84.6 \%$ of cases (11 of 13) showed complete apical closure at the last post-treatment visit. Our findings concerning survival and clinical success are in agreement with those of who reported the survival and clinical success of RET [6, 25-26] and closely followed by the investigations of those who reported the survival and clinical success of apexification [27-28]. It can be concluded that VIPT can effectively alleviate the clinical symptoms as RET and apexification, and resolution of radiographic pathology. 
Apexification, although it confers high degrees of clinical success, the root cannot develop physiologically [2-4], while RET was reported as a new treatment was able to realize root development. Lin, et al. [9] reported on the effectiveness of RET compared with apexification in root development (73.9\% vs $26.5 \%$ ). Decreasing apical diameter was found in our study: $92.3 \%$ of the treated teeth (12 of 13) showed at least a $20 \%$ decrease in apical diameter, and complete apical closure occurred in $84.6 \%$ of the treated teeth (11 of 13) at the last visit. The average decrease in apical diameter in individual cases from the preoperative radiograph to follow-up at 3,6 , and 12 months and last recall was respectively $7.98 \%, 24.1 \%, 70.3 \%$, and $91.0 \%$. In RET research, Li et al. [29] observed a change in apical diameter at 3 , 6 and 12 months was respectively $20.40 \%, 51.16 \%$ and $72.90 \%$. In the aspect of continued root lengthening, $38.5 \%$ of cases (5 of 13) met clinically meaningful within-case change [23] at 12 months. There was a $27.4 \%$ increase in root length at 12 months across all the cases in our study, compared with several clinical case series and retrospective studies in RET, which had reported radiographic changes in root length and reported the change of $4 \%-23.37 \%[6,24,25,27,29]$. It can be concluded that VIPT can effectively achieve the goal of root develop physiologically, and the results close to performance of RET, superior to apexification techniques.

The only case in this study that failed in resolving periapical lesions was one of dens evaginatus, having too severe apical periodontitis. Several researchers reported that Hertwig's epithelial root sheath and/or the apical papilla contributed to the recovery of the apical periodontitis and continued tooth development $[24,27]$. Therefore, too large a periapical radiolucency or root tip cyst should be cause for exclusion for VIPT. A case with an outstanding outcome in continuing tooth development was an 8-year-old boy with dens evaginatus in \#35 (Nolla 7) teeth who came to the clinic immediately (after fracture of the central cusp) having painful symptoms. The incident threw light on a histological feature of the pulp tissue of immature permanent teeth having the characteristics of a rich blood supply and high cell composition, which makes its regeneration and reparability much more feasible [12].

In conclusion, this study provides independent confirmation of the findings of VIPT by analyzing immature permanent teeth with irreversible pulp infection or periapical infection. The patients treated with VIPT became asymptomatic, no sinus tracts were evident, and the apical periodontitis was resolved. Moreover, there was radiographic evidence of root continuing development (apical closure and increased root length). Radiographic findings and clinical outcomes in immature teeth suggest that root canal exploration should be carried out when pulpitis in immature permanent teeth spreads to all pulps, even with periapical signs or symptoms. These residual vital tissues should be preserved as much as possible to regenerate a functional pulp-dentin complex. VIPT approaches can be an alternative option to treat immature teeth with irreversible pulpitis, even apical periodontitis.

There are several limitations to the present study. First, it is a retrospective case series evaluation, hence presents a lower level of evidence compared with a prospective randomized controlled trial. Nevertheless, this is the first retrospective study to observe the quantification of clinical and radiographic changes of the VIPT techniques. Second, the simple size was too small. In the published retrospective studies or case series, Sarah et al. [30] presented only 6 typical cases having different outcomes, whereas others case 
series presented 1 to 8 typical cases [17-21]. But all the pioneering efforts brought some suggestions for the clinical work and our findings shed light on the possibility of improving the clinical signs and symptoms and radiographic evidence of root development in VIPT. Therefore, prospective randomized clinical trials are needed to provide strong quantitative evidence for both treatment efficacy and the potential for adverse effects.

\section{Abbreviations}

\begin{tabular}{ll} 
Apx & Apexification \\
\hline VIPT & Vital inflamed pulp therapy \\
\hline RET & Regenerative endodontic treatment \\
\hline MTA & Mineral trioxide aggregate \\
\hline PARL & Periapical radiolucency
\end{tabular}

\section{Declarations}

\section{Ethical approval and consent to participate:}

This study received approval from the Office of Human Research Ethics at the Ninth People's Hospital, Shanghai Jiao Tong University, School of Medicine (Ref: SH9H-2019-T350-1). All procedures performed in studies involving human participants were in accordance with the ethical standards of the institutional research committee and with the 1964 Helsinki declaration and its later amendments or comparable ethical standards. Informed consent was obtained from all individual participants included in the study, and the written informed consent was obtained from a parent or guardian for participants under 16 years old.

\section{Consent for publication and Competing Interests:}

Wen Xiao declares that she has no conflict of interest. Wentao Shi declares that he has no conflict of interest. Jun Wang declares that she has no conflict of interest.

\section{Funding:}

This work was supported by the National Key R\&D Program of China (2017YFC0840100, 2017YFC0840110) (Prof. Jun Wang); the Interdisciplinary Program of Shanghai Jiao Tong University(YG2017QN04) (Dr. Wen Xiao) and Seeding Foundation of Shanghai Research Institute of Stomatology (2016-08) (Dr. Wen Xiao). 


\section{Authors' contributions:}

JW conceived the ideas; WX collected the data; WS analyzed the data; WX led the writing.

\section{Acknowledgements:}

The authors deny any conflicts of interest related to this study.

\section{References}

1. Endodontists. AAo. AAE Position Statement on the Scope of Endodontics: Regenerative Endodontics. Journal of Endodontics. 2013;39(4):561-3.

2. Witherspoon DESJ, Regan JD, et al. Retrospective Analysis of Open Apex Teeth Obturated with Mineral Trioxide Aggregate. Journal of Endodontics. 2008;34(10):1171-6.

3. RAMLM. T. Study of calcium hydroxide apexification in 26 young permanent incisors. Dent Traumatol. 2005;21(3):141-5.

4. Matsumoto T, Nakajima Y, Saito M, et al. Treatment of the immature tooth with a non-vital pulp and apical periodontitis. Dent Clin North Am. 2010;54(2):313-24.

5. Ree MHSR. Long-term Success of Nonvital, Immature Permanent Incisors Treated With a Mineral Trioxide Aggregate Plug and Adhesive Restorations: A Case Series from a Private Endodontic Practice. Journal of Endodontics. 2017;43(8):1370-7.

6. Nagy MM, Tawfik HE, Hashem AA, et al. Regenerative potential of immature permanent teeth with necrotic pulps after different regenerative protocols. Journal of Endodontics. 2014;40(2):192-8.

7. LP. CSC. Radiographic outcome of necrotic immature teeth treated with two endodontic techniques: A retrospective analysis. Biomed J. 2016;39(5):366-71.

8. II-Young JS-JL, Hargreaves KM. Biologically based treatment of immature permanent teeth with pulpal necrosis: a case series. Texas Dental Journal. 2012;129(6):876-87.

9. Jiacheng Lin QZ. Xi Wei. Regenerative Endodontics Versus Apexification in Immature Permanent Teeth with Apical Periodontitis: A Prospective Randomized Controlled Study. Journal of Endodontics. 2017;43(11):1821.

10. Jiang X, Liu H, Peng C. Clinical and Radiographic Assessment of the Efficacy of a Collagen Membrane in Regenerative Endodontics: A Randomized, Controlled Clinical Trial. Journal of Endodontics. 2017;43(9):1465.

11. Dabbagh BAE, et al. Clinical complications in the revascularization of immature necrotic permanent teeth. Pediatric dentistry. 2012;34(5):414-7.

12. W. S. Characterization of Apical Papilla and its Residing Stem Cells from Human Immature Permanent Teeth -A Pilot Study. Journal of Endodontics. 2008;34(2):166-71. 
13. Zanini MME, Simon S. Pulp Inflammation Diagnosis from Clinical to Inflammatory Mediators: A Systematic Review. Journal of Endodontics. 2017;43:1033-51.

14. Lin LSF, Skribner J. Pulp biopsies from the teeth associated with periapical radiolucency. Journal of Endodontics. 1984;10(9):436-48.

15. Huang TJSW, Liu Y, et al. The Hidden Treasure in Apical Papilla: The Potential Role in Pulp/Dentin Regeneration and BioRoot Engineering. Journal of Endodontics. 2008;34(6):645-51.

16. Ronald WKA, Shaul L, et al. Revascularization: a treatment for permanent teeth with necrotic pulp and incomplete root development. Journal of Endodontics. 2013;39(3):319-26.

17. Jung IYLS, Hargreaves KM. Biologically Based Treatment of Immature Permanent Teeth with Pulpal Necrosis: A Case Series. Journal of Endodontics. 2008;34(7):876-87.

18. Iwaya SIM, Kubota M. Revascularization of an immature permanent tooth with apical periodontitis and sinus tract. Dent Traumatol. 2001;17:185-7.

19. Ling-Huey Chueb GT-JH. Immature Teeth With Periradicular Periodontitis or Abscess Undergoing Apexogenesis: A Paradigm Shift. J Endod. 2006;32(12):1213.

20. Tsukiboshi M, Ricucci D, Siqueira, et al. Mandibular Premolars with Immature Roots and Apical Periodontitis Lesions Treated with Pulpotomy: Report of 3 Cases. Journal of Endodontics. 2017;43(9S):65-74.

21. Thomson A, Kahler B. Regenerative endodontics-biologically-based treatment for immature permanent teeth: a case report and review of the literature. Aust Dent J. 2010;55(4):446-52.

22. CM N. The development of the permanent teeth. J Dent Child 1960;4(254 - 66).

23. Saoud TM, Zaazou A, Nabil A, Moussa S, Lin LM, Gibbs JL. Clinical and radiographic outcomes of traumatized immature permanent necrotic teeth after revascularization/revitalization therapy. Journal of Endodontics. 2014;40(12):1946.

24. Alobaid AS, Cortes LM, Lo J, et al. Radiographic and Clinical Outcomes of the Treatment of Immature Permanent Teeth by Revascularization or Apexification: A Pilot Retrospective Cohort Study. Journal of Endodontics. 2014;40(8):1063-70.

25. Bose R, Nummikoski P, Hargreaves K. A retrospective evaluation of radiographic outcomes in immature teeth with necrotic root canal systems treated with regenerative endodontic procedures. Journal of Endodontics. 2009;35(10):1343.

26. Kahler B, Mistry S, Moule A, et al. Revascularization Outcomes: A Prospective Analysis of 16 Consecutive Cases. Journal of Endodontics. 2014;40(3):333-8.

27. Jeeruphan $T$, Jantarat $J$, Yanpiset $K$, et al. Mahidol study 1 : comparison of radiographic and survival outcomes of immature teeth treated with either regenerative endodontic or apexification methods: a retrospective study. Journal of Endodontics. 2012;38(10):1330.

28. Chen MY, Chen KL, Chen CA, et al. Responses of immature permanent teeth with infected necrotic pulp tissue and apical periodontitis/abscess to revascularization procedures. Int Endod J. 2012;45(3):294-305. 
29. Li L, Mei L, et al. Clinical and Radiographic Outcomes in Immature Permanent Necrotic Evaginated Teeth Treated with Regenerative Endodontic Procedures. Journal of Endodontics. 2017;43(2):24651.

30. Sarah Bukhari MRK, Frank Setzer, et al. Outcome of Revascularization Procedure: A Retrospective Case Series. Journal of Endodontics. 2016;42:1752-9.

\section{Figures}

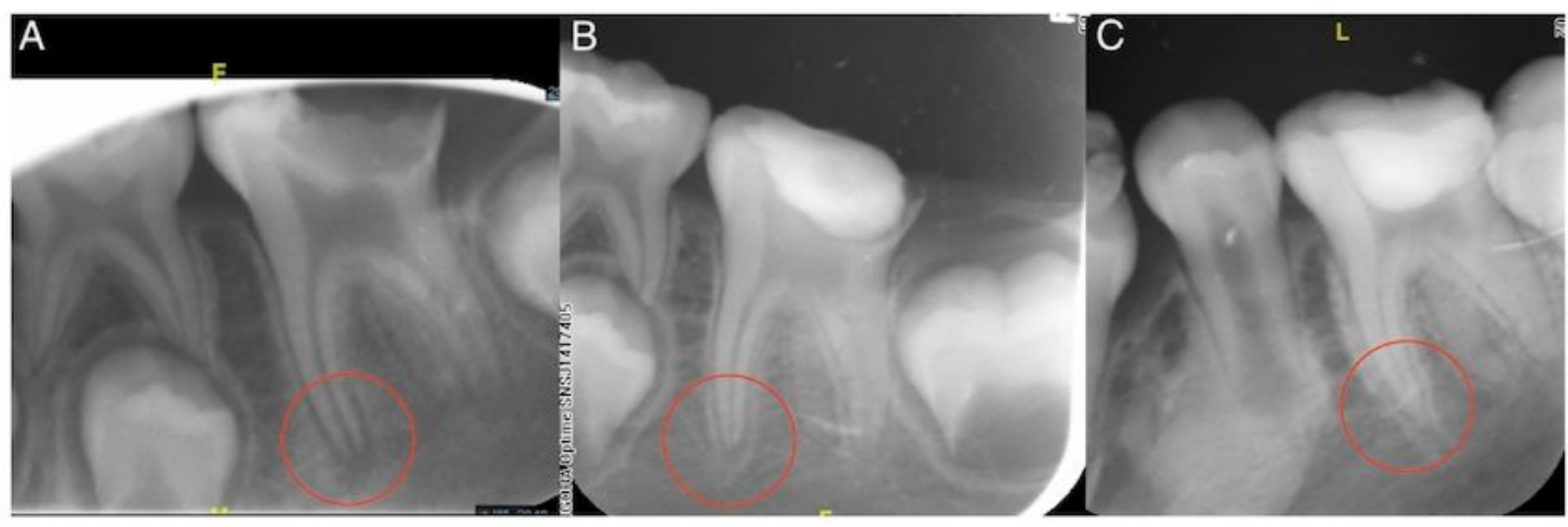

\section{Figure 1}

Complete healing category. Mandibular left first molor (\#36). Incresed thickening of the canal walls and continued root maturation after vital inflamed pulp therapy (VIPT). (A) Preperative radiograph. (B) Followup radiograph at 6 months. (C) Follow-up radiograph at 3 years

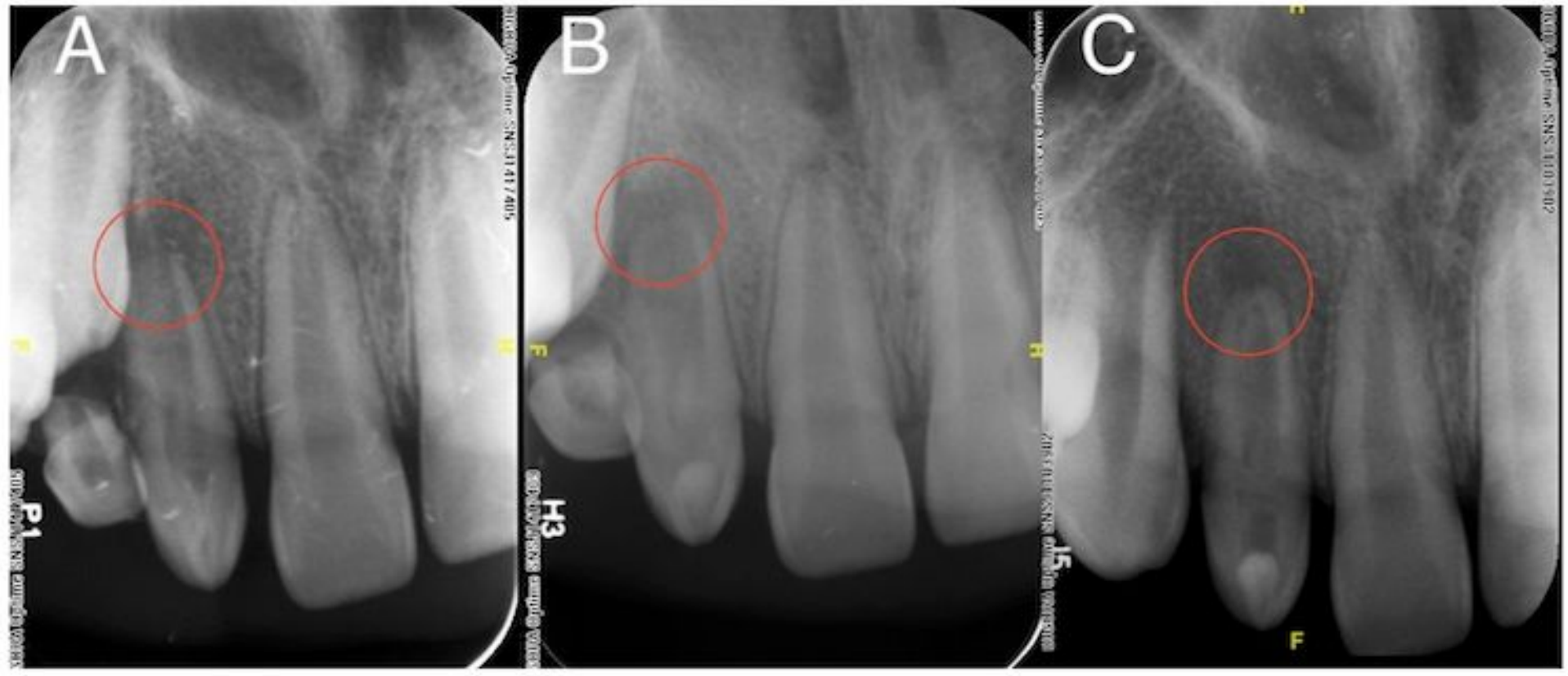

Figure 2 
Maxillary right lateral incisor (\#12) (A) Preperative radiograph of tooth with open apex and periapical radioluccency (B) Follow-up radiograph at 3 months (C) Follow-up radiograph at 18 months. Note continuous root extension and canaal wall thickening, relative to the preperative image.

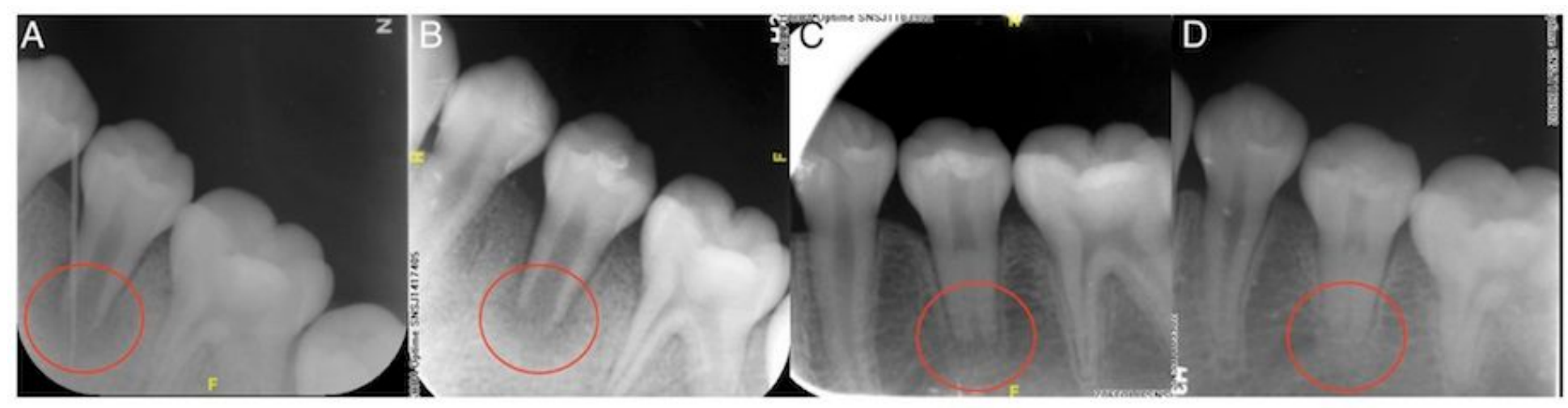

\section{Figure 3}

Mandibular left second premolar (\#35) (A) Preperative radiographic image showing an incompletely developed apex and a periapical radiolucency. Note the sinus tract that traces to the apex of this tooth.

(B) Follow-up radiograph at 3 months. (C) The radiograph demonstrating complete resolution of the radiolucency and continued development of the apex (calcification in root canal) at 18 months follow-up (D) Folow-up radiograph at 26 months
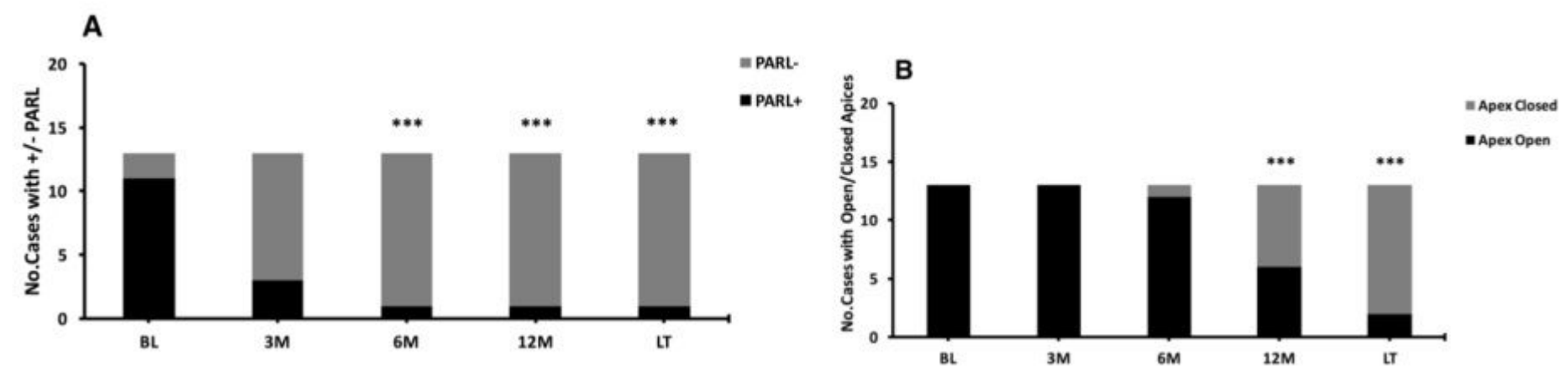

\section{Figure 4}

Radiographic findings regarding proportion of subjects with PARL and open apices at varios time points in the study. (A) Proportion of teeth with PARI significantly decresed by 6 months folow-up visit. (B) Proportion of subjects with open apex began to decrese and became significantly different from baseline at 12 months. ${ }^{\star \star \star} \mathrm{P}<0.05$ versus baseline BL-Baseline, LT- Last time recall 

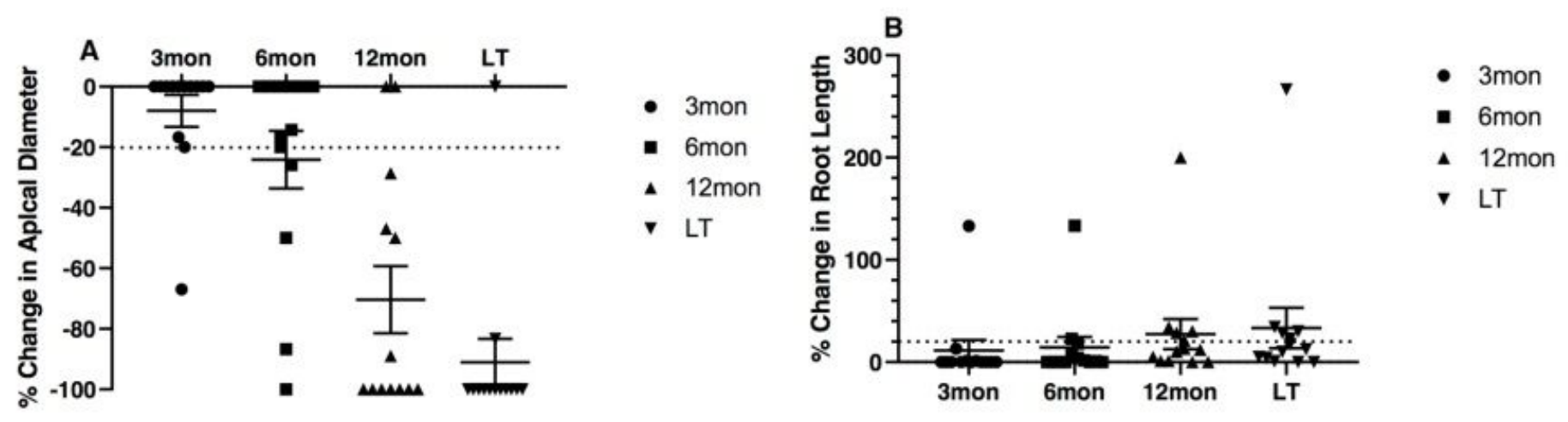

Figure 5

Scatter plot demonstrating the percentage change in neasured radiographic (A) apical diameter and (B) root length. Error bars represent meant standard error of the mean. Horizontal line denotes a $20 \%$ change, representing a clinically meaningful change. LT-LAst tome recall 